\title{
RESEARCH PAPER \\ MISSED OPPORTUNITIES FOR THE DIAGNOSIS OF PULMONARY TUBERCULOSIS IN ASHANTI AND WESTERN REGIONS OF GHANA; POLYMERASE CHAIN REACTION, A BETTER TOOL
}

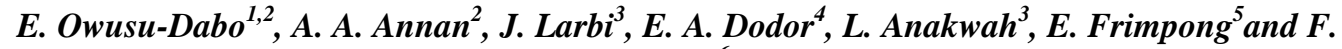 \\ Bonsu $^{6}$ \\ ${ }^{I}$ Department of Community Health, School of Medical Sciences, KNUST, Kumasi \\ E-mail: owusudabo@kccr.de \\ ${ }^{2}$ Kumasi Centre for Collaborative Research in Tropical Medicine, KNUST, Kumasi \\ ${ }^{3}$ Department of Theoretical and Applied Biology, KNUST, Kumasi \\ ${ }^{4}$ Communicable Disease Unit, Effia Nkwanta Hospital, Ghana Health Service, Takoradi \\ ${ }^{5}$ Department of Microbiology, Faculty of Allied Health Sciences, KNUST, Kumasi \\ ${ }^{6}$ National Tuberculosis Programme, Accra, Ghana
}

\begin{abstract}
The diagnosis of Pulmonary Tuberculosis (PTB) has mainly relied on sputum microscopy and culture. The use of molecular techniques such as the Polymerase Chain Reaction (PCR) and its overwhelming advantages compared with conventional diagnostic methodologies cannot be overemphasized. The main aim of this work was to compare the diagnostic sensitivities of microscopy, culture and PCR. Suspected PTB sputum samples were prospectively collected from six hospitals in the Ashanti and Western regions of Ghana. Microscopy was carried out on all samples at the field sites. Apart from culture and PCR, repeat sputum microscopy was carried out in the laboratories of the Kumasi Centre for Collaborative Research (KCCR). Out of the total 425 cases recruited for the study, 123 (29.0\%) were smear positive on site in contrast to $275(64.7 \%)$ positivity rate at KCCR. Regarding culture, 254 (59.9\%) samples were culture positive whilst PCR technique using INS $1 \& 2$ and PR $8 \& 9$ primers were positive in $59.9 \%$ and $56 \%$ of cases respectively. The proportion of missed positive cases of microscopy were $131(51 \%)$ compared with culture and $1.1 \%$ missed cases when compared with PCR. The sensitivity and specificity of microscopy were 123/254 (48.4\%) and 154/302 (50.99\%). The study confirms the superiority of $P C R$ in the diagnosis of PTB and indicates that a substantial proportion of PTB cases are missed when microscopy alone is used. In areas where the incidence of PTB is high and at referral hospitals, PCR can be done to augment the diagnosis of TB.
\end{abstract}

Keywords: Pulmonary Tuberculosis, Sputum Microscopy, Culture, Polymerase Chain Reaction

\section{INTRODUCTION}

Tuberculosis (TB) has become a global problem and the World Health Organization (WHO) estimates that globally 8.3 million cases of TB are notified each year. Of this, 2.6 million prove fatal, thus making TB the world's largest single infectious cause of death in spite of the widespread availability of highly effective 
drugs (Cock et al., 1994). The burden of the disease is mostly felt in developing countries, notably sub-Saharan Africa, where the HIV/ AIDS epidemic is the single most important factor responsible for the observed increase (WHO, 2002).

The recommended laboratory diagnosis of TB is based on examination of sputum after ZiehlNeelsen (ZN) staining for acid-fast bacilli (AFB) and culture. Although sputum smear microscopy efficiently identifies the most infectious cases, the effectiveness or otherwise of such method depends on the competence of the laboratory technician, the equipment used, and the quality of sputum produced by the patient as well as the immune status of the patient (Campbell and Bah-Sow, 2006). Moreover, ZN staining is not specific for Mycobacterium tuberculosis; it will be positive for any Mycobacteria species. Furthermore, the $\mathrm{ZN}$ staining technique has a detection limit of (5-10) $\times 10^{3}$ bacilli/ml and its sensitivity is between 40$60 \%$.

The use of molecular based techniques for diagnosing TB has been widely characterised and its superiority described (Andersen et al., 1993; Kolk et al., 1992; Noordhoek et al., 1994). However the issue of cost grossly hampers the introduction of these molecular based techniques in the management of cases with TB especially in developing countries (Floyd et al., 2006; Roos et al., 1998; Van Cleeff et al., 2005). The use of these methods in the diagnosis of TB may not only improve case detection but can also serve as a means of quality control mechanism for sputum smear microscopy.

The aim of this study was therefore to help identify what proportion of cases were missed routinely through sputum microscopy and to help situate the role of PCR in improving case detection for Ghana.

\section{MATERIALS AND METHODS}

Study Sites

The study was conducted at the Communicable
Diseases Unit of Effia-Nkwanta Hospital (CDU -ENRH) in the Western Region and the Chest Clinic of the Komfo Anokye Teaching Hospital (KATH), Kumasi South and the Agogo Presbyterian Hospitals, all in the Ashanti Region. These sites were chosen purposively based on their high case load and the conduct of DOTS in these sites.

\section{Study Population}

The study population included all patients with a history of cough for more than two weeks with any of the clinical manifestations of PTB (cough, weight loss, night sweats, fever etc.) and had sputum smear microscopy requested for the diagnosis of PTB.

\section{Sample size and Sampling Strategy}

All sites were purposively chosen on the basis of anticipated yield. A total of 1,410 samples from 425 eligible patients were collected during the period of the study. Each patient had to produce 3 sputum samples into $50 \mathrm{ml}$ sterile falcon tubes.

\section{Sputum Collection}

Trained technicians collected a sputum sample from each patient on the spot. Another sample was collected on day two and a third sample on day three. Direct smears for sputum microscopy were prepared and stained with the ZiehlNeelsen technique. The rest of the samples were transported to the Kumasi Centre for Collaborative Research (KCCR) for further analysis.

\section{Laboratory Procedures at KCCR \\ ZN staining}

Independent repeat microscopy was carried out on all sputum samples collected using the Ziehl -Neelsen (ZN) method.

\section{Examination of bacilli by ZN Staining}

Using a microscope, with oil immersion, the presence or absence of definite rod shaped AFBs was reported as positive and negative respectively using the WHO standard as shown below: 
PCR in the diagnosis of Pulmonary Tuberculosis ... 36

Table 1: Standard WHO microscopy grading for AFBs

\begin{tabular}{ll}
\hline Result & Grading \\
\hline More than 10 AFB / field for at least 20 fields & +++ \\
$1-10$ AFB / field & ++ \\
$10-99$ AFB / 100 fields & + \\
$1-9$ AFB / 100 fields & Marked with exact number \\
No AFB in at least 100 fields & - (Negative) \\
\hline
\end{tabular}

Source: www.who.int/entity/ihr/training/laboratory_quality/11_cd_rom_tb_eqa_wpro.pdf(assessed on March 11, 2008)

Culture of Bacilli

Samples were pre-treated with $4 \% \mathrm{NaOH}$ and $0.9 \% \mathrm{NaCl}$ for decontamination and concentrated by centrifugation. Following this step, $0.1 \mathrm{ml}$ of pellet suspension was inoculated into each of the two culture tubes containing Lowenstein-Jensen medium. The tubes were examined weekly for colonies. If any colony was seen at any stage, acid-fastness of the bacilli was determined by $\mathrm{ZN}$ staining. A portion of the remaining pellet was smeared on a slide, stained by $\mathrm{ZN}$ technique and examined. The remaining pellet was sent to the molecular biology laboratory for DNA extraction and downstream PCR. Negative report was given when no colonies appeared after observing weekly for 10 weeks.

PCR-Mycobacterial DNA extraction

DNA was extracted following the Qiagen DNA extraction protocol (Qiagen GmbH. Germany) according to the manufacturer's recommendation. Briefly, $200 \mathrm{ml}$ of sputum was lysed in a $1.5 \mathrm{ml}$ eppendorf tube after which the DNA was precipitated. The mixture was transferred onto a QIAamp Mini spin column and washed. Nucleic acids were eluted in a final volume of 200 $\mathrm{ml}$ of elution buffer.

\section{Amplification}

Amplifications were carried out on two different volumes of DNA $(2 \mathrm{ml}$ and $10 \mathrm{ml})$ by employing two sets of primers, INS1/2 and PR 8/9. Primers INS1 and 2 correspond to 631-650 and 856-875 respectively of the IS986 insertion element whilst PR8 and PR9 correspond to 105 -124 and 625-645 respectively of the IS986 insertion element.

Each amplification tube contained $1 \mathrm{ml}$ dNTPs (10mM each), $4 \mathrm{ml}$ of $\mathrm{MgCl}_{2}, 5 \mathrm{ml}$ of 10X PCR buffer, $1 \mathrm{ml}$ of primer each $(10 \mathrm{mM}), 0.25 \mathrm{ml}$ of Taq DNA polymerase and 27.75 and $35.75 \mathrm{ml}$ of RNAse free water for the $10 \mathrm{ml}$ and $2 \mathrm{ml}$ DNA volumes respectively.

Cycling conditions were 3 min initial denaturation at $95^{\circ} \mathrm{C}$, followed by 40 cycles of $1 \mathrm{~min}$ denaturation at $95^{\circ} \mathrm{C}, 1 \mathrm{~min}$ annealing at $60^{\circ} \mathrm{C}$ and $1 \mathrm{~min}$ extension at $72^{\circ} \mathrm{C}$. This was followed by $10 \mathrm{~min}$ final extension at $72^{\circ} \mathrm{C}$. Amplification was carried out in thermal cycler (Eppendorf, Germany).

Agarose gel electrophoresis and documentation Electrophoresis of the amplified products was done on a $1.5 \%$ ethidium bromide $(0.5 \mathrm{mg} / \mathrm{ml})$ stained agarose gel and visualized under a 254 nm UV transilluminator. The images were captured on a black and white polariod photographic film. A 550 base pair (bp) and a $220 \mathrm{bp}$ band when compared to a molecular weight marker were indicative of PR 8/9 and INS 1/2 respectively.

\section{Ethical clearance}

Research clearance was obtained from the Committee on Human Research Publication and Ethics, School of Medical Sciences, Kwame Nkrumah University of Science and Technology in Kumasi, Ghana.

\section{Data analysis}

Data were pooled across all sites for analysis. 
Descriptive statistics of the various sites from the data were analysed and presented in the form of tables. Using culture as the gold standard, proportions for the various tests were compared for both culture and PCR and are presented as percentages. Positive and Negative predictive values (PPV and NPV) for each test was calculated. All Statistical analyses were performed using SPSS 14.0.1 for windows (SPSS Inc. Chicago, USA).

\section{RESULTS}

Sputum microscopy, culture and PCR results A total of 425 patients were recruited for the study of which $245(57.6 \%)$ were males. Of these, approximately a quarter seen at the study sites were smear positive (123) of which $17.9 \%$ of them were $3+$. Comparatively, $64.7 \%$ of cases seen at KCCR were smear positive with $6.4 \%$ being $3+$. Culture results indicated that $59.8 \%$ were positive whilst PCR using primers INS $1 / 2$ and PR $8 / 9$ were positive in $59.1 \%$ and $56.0 \%$ respectively (Table 2 ).
Over an under-reading of cultures

In order to estimate the extent of over-reading, culture-negative specimens were taken as standards and compared with the results of corresponding microscopy at the study sites and KCCR. Overall, 171 specimens were negative by culture, $5.8 \%$ and $48.5 \%$ were reported by study sites and KCCR respectively as smear positive (Table 3).

Under-reading of culture-positive specimens To estimate the extent of under-reading at the study sites and KCCR, culture positive specimens were taken as standards and compared with results of the corresponding smears as reported by the laboratories. Out of the total of 254 culture positive samples, $44.1 \%$ and $75.6 \%$ were reported as smear positive by the study sites and KCCR respectively (Table 4).

Negative and Positive Predictive values Out of the total of 254 culture-positive samples, sputum smear microscopy detected 112, giving a sensitivity of $44.1 \%(112 / 254 * 100)$. On the

Table 2: Results of sputum microscopy, culture and PCR carried out

\begin{tabular}{llllll}
\hline Results & \multicolumn{2}{l}{$\begin{array}{l}\text { Microscopy No (\%) } \\
\text { Field }\end{array}$} & KCCR & Culture No (\%) & \multicolumn{2}{l}{$\begin{array}{l}\text { PCR No (\%) } \\
\text { INS1/2 }\end{array}$} & PR 8/9 \\
\hline Positive & $123(28.9)$ & $275(64.7)$ & $254(59.8)$ & $251(59.1)$ & $\begin{array}{l}238 \\
(56.0)\end{array}$ \\
Negative & $302(71.1)$ & $150(35.3)$ & $171(40.2)$ & $174(40.9)$ & $\begin{array}{l}187 \\
(44.0)\end{array}$ \\
Total & 425 & 425 & 425 & 425 & 425 \\
\hline
\end{tabular}

Table 3: Over-reading of sputum smears at the study sites and KCCR

\begin{tabular}{llll}
\hline Study sites & Total no. of culture- & \multicolumn{2}{l}{ Read as smear-positive at: } \\
& negative specimens & Study site & KCCR \\
\hline Agogo & 18 & $1(5.6 \%)$ & $9(50 \%)$ \\
KATH & 45 & $4(8.9 \%)$ & $18(40 \%)$ \\
Kumasi South & 40 & $1(2.5 \%)$ & $19(47.5 \%)$ \\
Takoradi & 68 & $4(5.9 \%)$ & $37(54.4 \%)$ \\
Total & $171(100 \%)$ & $10(5.8 \%)$ & $83(48.5 \%)$ \\
\hline
\end{tabular}


PCR in the diagnosis of Pulmonary Tuberculosis ... 38

Table 4: Under-reading of sputum smears at the study sites and KCCR

\begin{tabular}{llll}
\hline Study sites & Total No. of culture- & \multicolumn{2}{l}{ Read as smear-positive at: } \\
& positive specimens & Study site & KCCR \\
\hline Agogo & 25 & $19(76.0 \%)$ & $18(72.0 \%)$ \\
KATH & 148 & $52(35.1 \%)$ & $110(74.3 \%)$ \\
Kumasi South & 12 & $3(25.0 \%)$ & $6(50.0 \%)$ \\
Takoradi & 69 & $38(55.1 \%)$ & $58(84.1 \%)$ \\
Total & $254(100 \%)$ & $112(44.1 \%)$ & $192(75.6 \%)$ \\
\hline
\end{tabular}

other hand, out of the 171 culture-negative results, microscopy detected 161 , giving a specificity of $94.2 \%(161 / 171 * 100)$. Thus, the positive predictive value $(+\mathrm{PV})$ and negative predictive value (-PV) of smear microscopy is $44.1 \%$ and $94.2 \%$ respectively.

Smear microscopy compared with PCR

Out of the 251 specimens that were PCR positive (using INS $1 \& 2$ primers), sputum microscopy correctly identified 97 , giving a sensitivity of $38.6 \%(97 / 251 * 100)$. One hundred and seventy four (174) specimens were PCR negative, and microscopy correctly identified 149, a specificity of $85.6 \%(149 / 174 * 100)$. The $+\mathrm{PV}$ and -PV were $79.5 \%(97 / 122 * 100)$ and $49.2 \%$ $(149 / 303 * 100)$ respectively. When microscopy is compared with PCR, using PR $8 \& 9$ primers, of the 238 specimens that were PCR positive, microscopy correctly identified 101, with a sensitivity of $42.4 \%\left(101 / 238^{*} 100\right)$. Of the 187 specimens that were PCR negative, microscopy correctly picked 166, with a specificity of $88.8 \%(166 / 187 * 100)$. The +PV and -PV were $82.8 \%(101 / 122 * 100)$ and $54.8 \%$ $(166 / 303 * 100)$ respectively.

\section{DISCUSSION}

Missed cases from smear microscopy have constituted part of a growing debate about the reasons why global targets cannot be achieved. Our study has indicated that over half of the samples tested on the field purported to be negative constituted missed cases of potential
PTB. This was quite surprising since the global target for TB control under DOTS include detecting $70 \%$ of new smear positive cases by 2005 (case detection). Unfortunately, current estimates suggest that the global case detection is about 37\% (Dye et al., 2002). Apart from inherent weaknesses in the health system presenting challenges for improved case detection, missed diagnosis using sputum microscopy has also been reported (Baylan et al., 2004). Additionally, other problems such as the competence of the health worker, mechanical or optical faults of microscopes and availability of other materials in making the right diagnosis and the ability of the technician to adequately detect the presence of mycobacteria are all factors contributing to missed diagnosis (Addo $e t$ al., 2006).

Diagnosis of TB using microscopy alone in poor laboratory setting in developing countries such as Ghana has yielded sensitivity and specificity figures ranging from $53.1 \%$ to 99.8\% respectively (Levy et al., 1989). Garg et al. (2003) indicated that about $40-60 \%$ of patients with pulmonary disease and $75 \%$ of patients with extrapulmonary disease go undiagnosed by this traditional method. This means that quite a substantial percentage of patients are missed if one is to depend on this traditional method alone. The finding from this study agrees with Garg et al. (2003) that between 40$60 \%$ of patients with pulmonary TB are missed as a result of this technique. Since most health 
and diagnostic centres are ill-equipped in both capacity and equipment to handle the numerous sputum that get to them for diagnosis, it is necessary that some quality control mechanism is set in place for proof reading and ensuring that before sputum is declared as negative it is indeed negative. False negative is one big factor with smear microscopy leading to failure to improve case detection. Repeated reading in various sites will help avoid/reduce the number of false positive tests and improve case detection.

The superiority of PCR in terms of its sensitivity has been unparalleled by that of sputum microscopy. Several studies have underscored the importance of PCR in the diagnosis of tuberculosis (Dinnes et al., 2007; TrombertPaolantoni et al., 2006; Wang and Elston, 2007). This study shows that PCR as a technique greatly helped improve case detection. Unfortunately, WHO does not recommend the use PCR routinely due to associated cost implications especially in resource poor countries. It must be noted that even though PCR has a disadvantage of being expensive and requires highly skilled personnel to deliver the service as compared with that of microscopy some level of emphasis should be placed on it at least at referral hospitals to help improve case detection.

\section{CONCLUSION/ RECOMMENDATION}

This study has shown that a substantial number of TB cases are missed when smear microscopy alone is used in detecting AFBs. Molecular techniques show superiority in diagnosis and overwhelmingly improved case detection. Our study recommends among other things, the establishment of a local quality control system at the institutional level to provide reliability of microscopy. Secondly, establishment of a PCR "buffer system" to help supplement the work of field microscopy may also be needed.

\section{REFERENCES}

Addo, K. K., Owusu-Darko, K., Dan-Dzide, M., Yeboah-Manu, D., Ablordey, A., Caul- ley, P., Minamikawa, M., Bonsu, F., Lienhardt, C., Akpedonu, P. and Ofori-Adjei, D. (2006). Situation analysis of TB microscopy centres in Ghana. The International Journal of Tuberculosis and Lung Disease: the Official Journal of the International Union Against Tuberculosis and Lung Disease, 10 (8):870-875.

Andersen, A. B., Thybo, S., Godfreyfaussett, P. and Stoker, N. G. (1993). Polymerase ChainReaction for Detection of MycobacteriumTuberculosis in Sputum. European Journal of Clinical Microbiology \& Infectious Diseases, 12(12):922-927.

Baylan, O., Kisa, O., Albay, A. and Doganci, L. (2004). Evaluation of a new automated, rapid, colorimetric culture system using solid medium for laboratory diagnosis of tuberculosis and determination of anti-tuberculosis drug susceptibility. The International Journal of Tuberculosis and Lung Disease, 8(6) 772777.

Campbell, I. A. and Bah-Sow, O. (2006). Pulmonary tuberculosis: diagnosis and treatment. BMJ 20;332(7551):1194-7.

Cleeff, V. M., Kivihya-Ndugga, L., Githui, W., Nganga, L., Kibuga, D. and Odhiambo, J. (2005). Cost-effectiveness of Polymerase Chain Reaction versus Ziehl-Neelsen smear microscopy for diagnosis of tuberculosis in Kenya. International Journal of Tuberculosis and Lung Disease, 9(8):877-83.

Cock, K. M., Lucas, S. B., Kochi, A., Crofton, J., Glynn, J. R., Sterne, J. A. C., Rodrigues, L. C. and Maher, D. (1994). Global challenge of Tuberculosis. Lancet 344 (8922): 608609.

Dinnes, J., Deeks, J., Kunst, H., Gibson, A., Cummins, E., Waugh, N., Drobniewski, F. and Lalvani, A. (2007). A systematic review of rapid diagnostic tests for the detection of tuberculosis infection. Health Technology 
PCR in the diagnosis of Pulmonary Tuberculosis ... 40

Assess, 11(3):1-196.

Dye, C., Espinal, M. A., Watt, C., Mbiaga, C. and Williams B. G. (2002). Worldwide incidence of multidrug-resistant tuberculosis. Journal of Infectious Diseases, 185: 11971202.

Floyd, K., Arora V. K., Murthy, K. J. R., Lonnroth, K., Singla, N., Akbar, Y. (2006). Cost and cost-effectiveness of PPM-DOTS for tuberculosis control: evidence from India. Bulletin of the World Health Organization, 84(6):437-45.

Garg, S. K., Tiwari, R. P., Tiwari, D., Singh, R., Malhotra, D., Ramnani, V. K., Prasad, G. B. K. S., Chandra, R., Fraziano, M., Colizzi, V. and Bisen, P. S. (2003). Diagnosis of tuberculosis: available technologies, limitations, and possibilities. Journal of Clinical Laboratory Analysis, 17(5):155-63.

Kolk, A. H. J., Schuitema, A. R. J., Kuijper, S., vanLeeuwen, J., Hermans, P. W. M. and van Embden, J. D. A. (1992). Detection of Mycobacterium-Tuberculosis in Clinical-Samples by Using Polymerase Chain-Reaction and A Nonradioactive Detection System. Journal of Clinical Microbiology, 30(10):2567-75.

Levy, H., Feldman, C., Sacho, H., Meulen, H., Kallenbach, J. and Koornhof, H. (1989). A re -evaluation of sputum microscopy and cul- ture in the diagnosis of pulmonary tuberculosis, 95(6):1193-1197

Noordhoek, G. T., Kolk, A. H. J., Bjune, G., Catty, D., Dale, J. W. and Fine, P. E. M. (1994). Sensitivity and Specificity of PCR for Detection of MycobacteriumTuberculosis - A Blind Comparison Study Among seven Laboratories. Journal of Clinical Microbiology, 32(2):277-84.

Roos, B. R., vanCleeff, M. R. A., Githui, W. A., Kivihya-Ndugga, L., Odhiambo, J. A. and Kibuga, D. K. (1998). Cost-effectiveness the polymerase chain reaction versus smear examination for the diagnosis of tuberculosis in Kenya: a theoretical model. International Journal of Tuberculosis and Lung Disease 2 (3):235-41.

Trombert-Paolantoni, S., Figarella, P. and Clairet, V. (2006). Contribution of PCR for detection of Mycobacterium tuberculosis complex in respiratory and nonrespiratory specimens. Pathol Biol, 54(8-9): 488-92.

Wang, T. and Elston, R. C. (2007). Improved power by use of a weighted score test for linkage disequilibrium mapping. Am J Hum Genet, 80(2):353-360.

WHO Press Release. (2002). HIV causing tuberculosis cases to double in Africa 122. Tropical Doctor; 32(3):188. 